\title{
Protetik tedavilerde lazerlerin kullanımı
}

\author{
Yusuf Ziya Akpınar ${ }^{1}$, Önjen Tak², Özlem Koç3 , Aslıhan Üşümez
}

Selcuk Dental Journal, 2015; 1: 33-42

Başvuru Tarihi: 22 Şubat 2015 Yayına Kabul Tarihi: 04 Mart 2015

\begin{abstract}
The use of lasers in prosthetic treatment
The aim of this study was to discuss how to integrate laser technology into the prosthetic treatment and effects on dental materials used currently in dental treatments. This article comprises scientific articles in the literature regarding as laser types in used prosthetic treatment, laser parameters and interactions between laser and dental materials.
\end{abstract}

\section{KEY WORDS}

Dental material, laser, prosthetic treatment, surface treatment

LASER kelimesi "Light Amplification by Stimulated Emission of Radiation" kelimelerinin ilk harflerinden oluşmaktadır. Dilimize "LAZER" olarak çevrilmiş bu kelime" Radyasyonun uyarılmış emisyonu ile ışığın güçlendirilmesi" anlamına gelmektedir.(Doğan ve ark 2014) Lazer sistemlerinin çalışma teorisi 1960 yilında Albert Ainstain tarafından ortaya konan Işıma teorisine (Stimulated Emission) dayanmaktadır. Bu teoriye göre bir atom veya molekülün fotonla uyarılması sonucunda düşük enerji seviyesindeki (Ed) elektron yüksek enerji (Ey) seviyesine geçerek (Soğurma= Absorpsiyon) atomu kararsız hale getirir (Şekil 1a). Atomun tekrar kararlı hale gelmesi için Ey seviyesindeki elektron tekrar Ed seviyesine geçmesi gerekir. Bu geçiş sırasında atom kendisini uyaran fotonla aynı dalga boyuna sahip ikinci bir fotonun
$\left(\mathrm{E}_{\text {foton }}=\mathrm{h}\right.$ ) $)$ saçılmasına neden olur. Böylelikle bir atomdan uyarılma (excitation) ile beraber iki foton daha saçılmış (emisyon) olur (Şekil 1b).

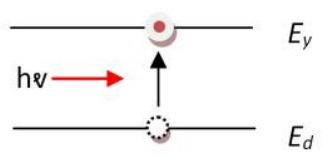

a

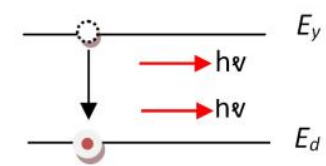

b
Şekil 1.

a Atomun uyarılması sonucu elektronun düşük enerji seviyesinden yüksek enrji seviyesine geçmesi

b Yüksek enerji seviyesindeki elektronun tekrar eski seviyesine geçmesi

Sayıları giderek artan fotonlar bir oda içerisinde biri yarı geçirgen diğeri tam yansıtıcı olmak üzere (optik resonator) iki ayna tarafından doğrultuları (collimated) ve aynı fazda (koherans) ve aynı dalga boyunda (monochromatik) dışarıya çıkarak lazer demetini (laser beam) oluştururlar (Şekil 2).

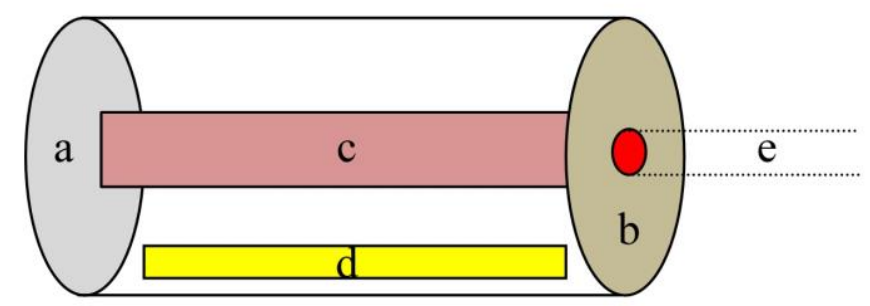

Şekil 2.

Katı hal lazerin yapısı; (a) tam yansıtıcı ayna, (b) yarı geçirgen ayna, (c) kristal, (d) uyarıcı lamba, (e) lazer ışın demeti

\footnotetext{
${ }^{1}$ Abant İzzet Baysal Üniversitesi Diş Hekimliği Fakültesi Protetik Diş Tedavisi Anabilim Dalı, Bolu

${ }^{2}$ Kocaeli Üniversitesi Diş Hekimliği Fakültesi Protetik Diş Tedavisi Anabilim Dalı, Kocaeli

${ }^{3}$ Bezmialem Vakıf Üniversitesi Diş Hekimliği Fakültesi Protetik Diş Tedavisi Anabilim Dalı, İstanbul
} 
Lazer sistemleri aktif mediumda bulunan kristal yapının fiziksel ve kimyasal özelliklerine göre farklı tiplere ayrılırlar (Örn: Neodymiumdopedyttriumaluminiumgarnet (Nd:YAG), Erbiumdopedyttriumaluminiumgarnet (Er:YAG)). Bu özellik lazerin farklı sahalarda kullanılabilmesine olanak sağlamaktadır. Diş hekimliği alanında ilk defa lazer kullanımı 1964 yılında Stern ve Sognnaes tarafından gerçekleştirilmiştir (de Andrade ve ark 2008). Lazerin diş hekimliğine girişinden hemen sonra sadece kullanımı değil, aynı zamanda kullanım sahası da giderek genişlemiştir (Eltas ve Orbak 2013).

\section{Lazerin protetik tedavilerde kullanılan materyaller üzerindeki etkisi}

\section{Seramik Yüzey İşlemleri}

Tamamı seramik restorasyonlar sahip oldukları üstün biyomekanik ve estetik özelliklerinden dolayı diş hekimlerinin yoğun ilgisini çekmiştir (Piconi ve Maccauro 1999). Bununla birlikte bazı araştırmacılar tamamı seramik kron ve köprülerin tutuculuklarını, kenar uyumlarını ve kırılma dirençlerini geliştirmek için değişik fikirler ortaya atmışlardır (Atsu ve ark 2006).

\subsection{Rezin siman bağlantısı için mikro pörözitenin oluşturulması}

Indirekt restoratif prosedürlerin klinik başarısı, restorasyon ve diş dokuları arasındaki bağlantıyı sağlamak için kullanılan simantasyon tekniğine bağlıdır (Radovic ve ark 2008). Geleneksel simanlarla karşılaştırıldığında, rezin simanların mekanik ve adeziv özelliklerinin yüksek olması onların daha fazla ilgi görmesine neden olmuştur (Kitasako ve ark 2001, Attar ve ark 2003). Rezin simanla seramik materyal arasındaki bağlantı dayanımı, seramik materyalin kimyasal kompozisyonuna, silan kaplama ajanına ve seramik üzerinde yapılan yüzey işlemlerine bağlıdır.

Diş hekimliğinde kullanılan restoratif materyallerin bağlantı mekanizması iki temel teori üzerine dayanmaktadır. Bunlardan birincisi kimyasal adezyon, diğeri ise mikro mekanik tutuculuk (Martínez-Insua ve ark 2000). Seramik yüzey üzerinde yapılan yüzey işlemleri bağlantı için gerekli yüzey alanının artmasına ve yüzeyde mikro pöröziteler yaratarak simanın mikromekanik bağlantısının artmasına neden olmaktadır (Borges ve ark 2003). Seramik restorasyon ile diş dokuları arasındaki bağlantı dayanımının artması tutuculuğun ve restorasyonun kırılma direncini arttırırken mikro sızıntıyı da azaltmaktadır (Blatz 2003).

\subsection{Alüminus seramik restorasyonlar}

Yüksek alümina içerikli seramik materyallerin (alumina based ceramics) yüksek stress taşıma (high-stress-bearing) özelliğinde dolayı tamamı seramik restorasyonlar içerinde büyük ilgi görmüştür (Guazzato ve ark 2005). Bu tür restorasyonlara örnek olarak gösterilen In-Ceram Alumina yüksek oranda alümina (82\%) ve az miktarda silica (4.5\%) içerir. Günümüz literatüründe bu türden restorasyonların yüzey pürüzlülüğü arttırmak için değişik yöntemler tarif edilmiştir (Sorensen ve ark 1990, Aida ve ark 1995). Bunların bazıları: 1Rocatec ve Silicoater, 2- Nd:YAG lazer, 3Er:Cr;YSGG lazer, 4- Nd:YAG lazer + Rocatec Sistem, 5- $\mathrm{Al}_{2} \mathrm{O}_{3}$ Sandblasting, Nd:YAG lazer, silane uygulaması ve kombinasyonları (Kern ve Thompson 1994, Miserendino 1995, Da Silveira ve ark 2005 , Osorio ve ark 2010, de Paula Eduardo ve ark 2012).

$\mathrm{Al}_{2} \mathrm{O}_{3}$ kumlama ve Rocatec Sistem, Er:Cr;YSGG lazer ile kıyaslandığında her üç yönteminde mikrotensile bağlantı dayanımları benzer bulunmuştur (de Paula Eduardo ve ark 2012). Bununla birlikte Nd:YAG lazer kullanımı rezin siman bağlantı dayanımını anlamlı derecede arttırmaktadır (Da Silveira ve ark 2005).

Karbon dioksit lazerin $\left(\mathrm{CO}_{2}\right.$ lazer) dalga boyunun neredeyse tamamı seramik materyaller tarafından absorbe edilir. $\mathrm{Bu}$ nedenle $\mathrm{CO}_{2}$ lazerler bu tür malzemelerin yüzey işlemlerinde etkili bir şekilde kullanılabilir. Ancak işlem sırasında açı̆̆a çıkan yüksek ısı yüzeyde conchoidal kırıklara neden olur (Dobberstein ve ark 1989). Diğer bir çalışmada ise kıyaslandığında cam infiltre edişmiş alimuna seramiklerde $\mathrm{CO}_{2}$ lazerin bağlantı dayanımı üzerine etkisi geleneksel yüzey pürüzlendirme işlemleriyle benzer bulunmuştur (Ersu ve ark 2009).

\subsection{Feldspathic seramik restorasyonlar}

Metal desteksiz feldspathic seramik restorasyonlar (onlay, inlay, lamine veneerler ve tam seramik kronlar) estetik özelliklerinin metal destekli restorasyonlara kıyasla daha üstün olmasından dolayı popüler olmuşlardır (Stewart ve ark 2002, Shiu ve ark 2007). Ancak bu tür restorasyonların mekanik özellikleri oldukça zayıf olup makaslama kuvvetleri karşısında sıklıkla kırılabilmektedirler (Jensen ve ark 1989, Burke 1995). Ayrıca bu tür restorasyonların diş dokularıyla olan bağlantıları zayıf olduğu için rezin simanlara intiyaç duyarlar (Fradeani ve Redemagni 2002).

Bu tür restorasyonların mikromekanik tutuculuğunu arttırmak için farklı yüzey işlemleri tanımlanmıştır (Da Silveira ve ark 2005, Shiu ve ark 2007, Akyıl ve ark 2010b, Akyıl ve ark 2011, Kara ve ark 2011). 
Hidroflorik asit (HF) uygulaması, $\mathrm{Al}_{2} \mathrm{O}_{3}$ kumlama, Er:YAG lazer, Nd:YAG lazer ve bunların kombinasyonunu içeren uygulamalar bulunmaktadır. $\mathrm{Bu}$ yöntemler içerinde en iyi bağlantı değeri HF asit ile sağlanmıştır. Ancak, Er:YAG yada Nd:YAG lazerin tek başına kullanılması rezin simanın bağlantı dayanımını olumsuz yönde etkilemiştir (Shiu ve ark 2007, Akyıl ve ark 2010b). Bununla birlikte lazerin HF den önce kullanıması rezin simanın feldspathic porselen olan bağlantı dayanımını arttırmaktadır (Akyll ve ark 2011).

\subsection{Lityumdisilikat seramik restorasyonlar}

Bu tür restorasyonlarda Er:YAG lazer kullanımı (300 $\mathrm{mj}, 600 \mathrm{mj}$ ve $900 \mathrm{mj}$ ) materyal içerisindeki lösit kristallerinde düzensizliklere neden olduğu bildirilmiştir. Bununla birlikte lazerin çıkış gücü arttıkça bağlantı değerinin azaldığı vurgulanmıştır. $\mathrm{Bu}$ nedenle bu tür restorasyonların SBS değerlerini arttırmak için yüksek enerji değerlerinden kaçınmak gerekir (Gökçe ve ark 2007). Er:YAG lazer seramik yüzeylerde \%43 oranında transmisyona uğradığı için bu tür lazerlerin seramik yüzeyleri üzerindeki pürüzlendirme etkisi tartışmalıdır (Morford ve ark 2011).

\subsection{Seramik restorasyonların çıkartılması (Debonding)}

Lazer debonding tekniği ilk olarak 1990 yilında seramik braketlerin sökülmesi için kullanılmıştır (Azzeh ve Feldon 2003). Bu amaç doğrultusunda CO2 lazer, Nd:YAG lazer, diod lazer, ytterbium fiber lazer ve Er:YAG lazer kullanılmıştır (Rickabaugh 1994, Ma ve ark 1997, Feldon ve ark 2010, Sarp ve Gülsoy 2011, Mundethu ve ark 2013). Lazer tipleri kadar kullanılan seramik türü de debonding işlemi için önemlidir; özellikle bu tür işlemler için monokristal yapıya sahip seramik braketler tercih edilmektedir (Azzeh ve Feldon 2003). Tocvhio ve ark (1993) bu durumu Isısal yumuşama (thermal softening), ısıyla patlama (thermal ablation) ve ışıkla patlama (photo ablation) olarak açıklamaktadır.

Lazer debonding sadece braketlerin sökülmesinde değil aynı zamanda kompozit dolguların diş dokularına zarar vermeden etkili bir şekilde sökülmesinde de etkili bir yöntemdir (Morford ve ark 2011). Öztoprak ve ark (2012) lityum disilikat laminate veneerlerin Er:YAG lazer ile kısa sürede (39 s) sökülebildiğini bildirmiştir.

\subsection{Lazer ile bağlantı dayanımının arttırıması}

Silan kaplı porselen yüzeyleri $\mathrm{CO}_{2}$ lazer $(10.600 \mathrm{~nm}$ dalga boyu, $1 \mathrm{~W}$, devamlı dalga modu (cw), $30 \mathrm{~mm}$ mesafeden) ile ışınlandıkları zaman kompozit rezin ile porselen arasındaki bağlantı artmaktadır. Bununla beraber yüzey pürüzlülük değerleri $\mathrm{Al}_{2} \mathrm{O}_{3}$ kumlama
(50 $\mu \mathrm{m}, 15 \mathrm{~s}, 0.2 \mathrm{MPa}$ basınç, $20 \mathrm{~mm}$ uzaklık) ile kıyaslandığında istatistiksel olarak anlamlı fark bulunmamıștır (Chen ve ark 2010). Sonuç olarak seramik yüzeyi üzerine uygulanan silan ajanlarının lazer ile ışınlanması porselen tamirinde etkili bir yöntem olarak kabul görmektedir.

\section{Zirconia yüzey pürüzlendirmesi}

Yttrium stabilized tetragonal zirconia'nin (Y-TZP) gelişimi diş hekimliğinde kullanılan seramikler içerinde yeni bir sınıfın doğmasına neden olmuştur (Lüthy ve ark 2006). Yüksek dayanım direncine sahip bu materyalin seramiklerde olduğu gibi diş hekimliğinde de oldukça geniş bir kullanım alanına sahiptir (Wolfart ve ark 2007, Özcan ve ark 2008). YTZP 'nin diğer seramiklere göre farklı kılan özelliği ise stress ile tetiklenen şekil değiştirme dayanıklılı̆ına (strees-induced transformation toughtening) sahip olmasıdır. Bu özelliği sayesinde zirconia materyali yüksek stressler altında mikrokristal yapısını değiştirerek stresslere karşı daha dayanıklı hale gelmektedir (Piconi ve Maccauro 1999, Guazzato ve ark 2004). Y-TZP normal koşullar altındaki flexural dayanıklıı̆ı 700-1200 MPa arasındadır. $\mathrm{Bu}$ değerler çiğneme sırasında meydana gelecek okluzal kuvvetleri fazlasıyla karşllayabilecek değerdedir (Yilmaz ve ark 2007). Bununla birlikte Y-TZP'nin kimyasal kompozisyonunu bağlantı dayanımını olumsuz yönde etkilemektedir (Kern ve Wegner 1998). Bu olumsuzlukların giderilmesi için Y-TZP'nin bağlantı yüzeyinde aşağıdaki modifikasyonları yapılması tavsiye edilmektedir (Blatz ve ark 2004, Spohr ve ark 2008, Cavalcanti ve ark 2009, Akyıl ve ark 2010a, Ural ve ark 2010, Akin ve ark 2011, Foxton ve ark 2011, Paranhos ve ark 2011, Subaşı ve Inan 2011, Demir ve ark 2012, Ural ve ark 2012, Usumez ve ark 2012, Akpinar ve ark 2015); Al2O3 kumlama, Er:YAG lazer, $\mathrm{Nd}$ YYAG lazer, $\mathrm{CO}_{2}$ lazer, Femto saniye lazer.

Nd:YAG lazer zirconia yüzeyini pürüzlendirerek rezin simanın shear bond strength (SBS) değerinin anlamlı derecede arttırmaktadır (Paranhos ve ark 2011). Üşümez ve ark (2012) yapmış oldukları bir çalışmada uzun atımlı (long pulse) ve kısa atımlı (short pulse) Nd:YAG lazerlerin SBS değerini anlamlı derecede arttırdığını ancak Nd:YAG lazerin mikroskobik görüntülerde zirconia yüzeyinde mikro çatlaklar oluşturduğunu bildirmiştir (Şekil 3).

Er:YAG lazer kontrol gruplarıyla karşılaştırılığında bağlantı değerlerini arttırırken siman-seramik arayüzündeki mikrosızıntı değerlerini azalttığı bildirilmiştir (Akin ve ark 2011). Subaşı ve ark (2011) yapmış olduğu bir çalışmada $400 \mathrm{mj}$ Er:YAG lazer kullanımı zirconia yüzeyinde az sayıda çukurcuk oluşumuna neden olduğunu vurgulamıştır. 


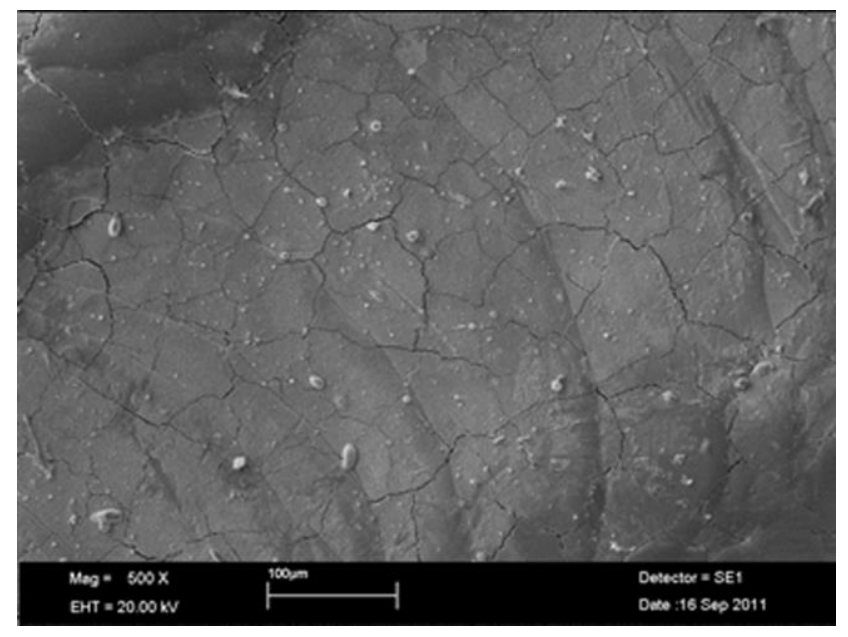

Şekil 3.

Nd:YAG lazerin Y-TZP yüzeyinde oluşturduğu mikro çatlaklar

Literatürde zirconia restorasyonlar için $\mathrm{CO}_{2}$ lazer kullanımı tartışıla gelmiştir. Ural ve ark (1995) 3w $\mathrm{CO}_{2}$ lazer kullanımının rezin simanın bağlantı dayanımını arttırdığını ifade etmiştir. Bunun aksine Paranos ve ark (2011) 5w CO lazer kullanımının yüzeyde çatlaklar oluşturduğunu ve SBS değerlerini azalttığını vurgulamıştır.

Mühendislik alanında kullanımı 1980'lere dayanan femto saniye lazerlerin diş hekimliği alanında kullanımı giderek artmaktadır. Bu tür lazerlerin atım sürelerinin (pulse duration) muazzam derecede kısa olması (10-15 s) atım başına düşen enerji miktarını arttırırken oluşturduğu ısı miktarı da benzer şekilde azalmaktadır. Akpinar ve ark (2015) yapmış olduğu çalışmada femto saniye lazerle Y-TZP yüzeyinde farklı yüzey geometrilerinin ve şekillerinin SBS değerleri üzerine etkisi karşılaştırmış ve sonuçta farklı geometriler arasında anlamlı fark olmadığını ancak çıkıntılı yüzeylerdeki bağlantı değerlerinin girintili yüzeylerden daha yüksek olduğunu bulmuşlardır. Ayrıca aynı çalışmada Y-TZP yüzeyinde femto saniye lazerle yapılan işlemelerin yüksek hassasiyette olduğu, yüzeyde mikro çatlakların olmadığı ve $X$ ışını kırınım cihaz (XRD) verilerine göre faz değişimi göstermediği belirtilmiştir (Şekil 4).

\section{Metal Yüzey İşleme}

\subsection{Titanyum}

Restoratif diş hekimliğinin ilgi çeken materyallerinden bir diğeride titanyumdur. (Fenton 1996) Titanyumun biyouyumluluğu, korozyona karşı gösterdiği yüksek direnç ve diğer metallere göre özgül ağırlığının çok düşük olmasından dolayı özellikle implant ve metal destekli seramik restorasyonlar için ideal bir materyal olarak tercih edilmiştir (Oshida ve Hashem 1993, Cai ve ark 2003, Östman ve ark 2005). Ancak seramik materyalin fırınlanması sırasında oluşan oksit tabaka

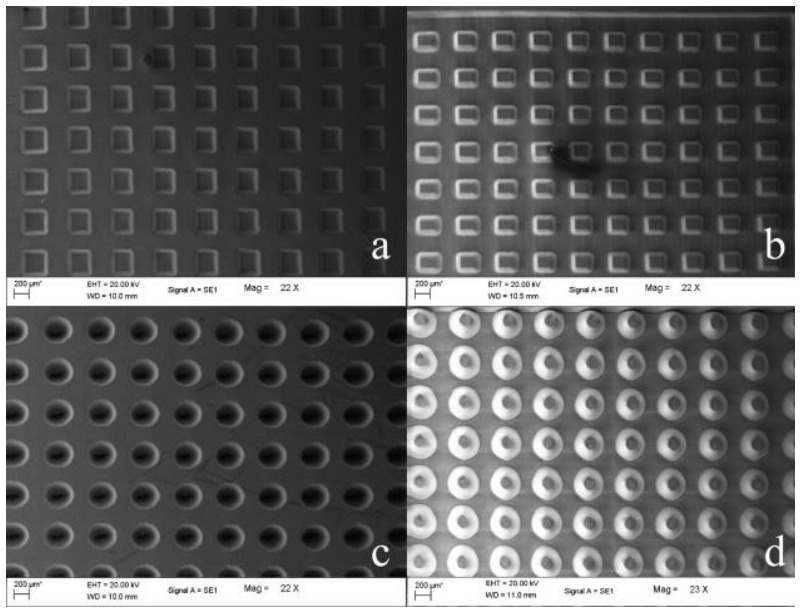

Şekil 4.

Femto saniye lazerin Y-TZP yüzeyinde oluşturduğu hassas mikro geometrik yapılar:

a kare girinti

b kare çıkıntı,

c yuvarlak girinti

d yuvarlak çıkıntı

metal-porselen bağlantısını olumsuz yönde etkilemektedir (Adachi ve ark 1990). Bu tabaka asit ile dağlama ve kumlama yöntemi ile kontrol edilebilmektedir (Hautaniemi ve ark 1992, Reyes ve ark 2001). Kim ve Cho (2009) geleneksel yöntemlere alternatif olarak Nd:YAG lazer kullanımının porselen-titanyum bağlantısını anlamlı derecede arttırdığını bildirmiştir.

\subsection{Değersiz metal alaşımlar}

Değersiz metal alaşımların ile akrilik rezinler arasındaki bağlantının zayıf olması her iki materyalin ısısal genleşme katsayılarının ve kimyasal kompozisyonlarının farklı olmasına dayanmaktadır. Bu farklılık protezlerde renklenmelere, kötü kokulara, akrilik rezinde kırılmalara ve patojenik mikro organizmaların birikimine neden olur (Creugers ve ark 1990, Barclay ve ark 2007). Bu nedenle hareketli bölümlü protezlerde (HBP) metal alaşımın pürüzlendirmesi gerektiği vurgulanmıştır (Marinello ve ark 1987). Konuyla ilgili literatürler incelendiğinde metal kaide ile akrilik rezin arasındaki bağlantıyı güçlendirmek için Nd:YAG. $\mathrm{XeCL}$ ve Er:YAG lazerlerin kullanıldığı görülmüştür (Murray ve ark 2005, Yilmaz ve ark 2011, Kunt ve ark 2012). Yilmaz ve ark (Yilmaz ve ark 2011) yürüttüğü bir çalışmada Nd:YAG lazer kullanımı Co-Cr materyali ile polymethylmethacrylate materyali arasındaki bağlantı dayanımını arttırdığını bildirmiştir. 


\section{Kaynak( Welding)}

Metal parçaların - doldurucu materyal olsun ya da olmaksızın - ISı yardımıyla birleştirilme işlemidir (Wulfes 2003). Lazer ile kaynak geleneksel yöntemlere göre (açık veya kapalı kaynak tekniği) birçok avantaj sunmaktadır (Sjögren ve ark 1988, Jemt ve Linden 1991, Dominici ve ark 1995, Chai ve Chou 1998, NaBadalung ve Nicholls 1998, Jemt ve ark 2000, Wiskott ve ark 2001, Liu ve ark 2002, Santos ve ark 2003, Zupancic ve ark 2006, Fasbinder 2010):

- Bağlantı kısmının daha güçlü olması

- Lokalize ve minimal ısı oluşturması (1-2 mm)

- Lokalize ısısının akrilik rezine ve seramik materyale ulaşmaması

- Revetman model ve model transferi gerektirmemesi

- Alt yapımım maksimum hassasiyette ve minimum distorsiyonla birleşmesi

- Co-Cr alaşımlarda lehimlenen bölgenin korozyona uğramaması

- İşlemin hızlı ve basit olması

Yukarıda sayılan avantajların yanında lazer kaynağın olumsuz yanları da bulunmaktadır: Cihazın pahalı olması (7200 \$), seramik materyalin $\mathrm{Ti}$ ve Ni-Cr-Mo esası alaşımlara bağlantı dayanımını zayıflatması (Aladag ve ark 2011, Galo ve ark 2011). Kaynak işlemi sırasında dikkat edilmesi gereken noktalardan biri enerji penetrasyon derinliğinin iyi ayarlanması; diğeri ise kaynak şeklinin dairesel olması; öyle ki her bir lazer dalgasının $\% 80$ dairesel olarak bir öncekinin üzerine süperpoze olmalıdır (Bertrand ve ark 2001). Kaynak derinliği lazer parametrelerine ve kullanılan metale göre değişkenlik gösterebilir. Ancak bu değer 2 mm' yi geçmemelidir (Lin ve ark 2007, Shimakura ve ark 2009). Titanyum gibi çabuk oksitlenebilen metaller için kaynak işlemi sırasında oksitlenmeyi engelleyici Argon (Ar) gazı kullanılabilir (Prasad ve Monaco 2009). Metal çiftleri arasında meydana gelen kaynağın kalitesi cihazın gerilimine (Volt ), lazer atım uzunluğuna (pulse length), lazer ışı demetinin çapına (beam diameter) ve enerji yoğunluğuna bağlıdır (joul/cm2) (Suzuki ve ark 2004). Suzuki ve ark (Suzuki ve ark 2004), kırımış kroşe kolunun ticari saf titanyum iskelet kaidesinin kaynatımasına 180 Volt, $3 \mathrm{~ms}$ atım süresi, $0.6 \mathrm{~mm}$ spot çapına sahip lazer kullanmıştır.

\subsection{Ağız içi lazer kaynağı (Intraoral laser welding)}

Lazer teknolojisinin getirdiği avantajlardan bir diğeri ise ağız içi lazer kaynağıdır (Fornaini ve ark 2011). Ağız içi lazer kaynağı, günlük klinik hayatımızda yaşadığımız problemleri etkili ve hızlı bir şekilde çözümlenmesine yardımcı olur (Fornaini ve ark 2009). Tam ağız restorasyonlarda, bar tutuculu protezlerde ve splint kronlarda yaşanan balans problemlerinin çözülmesine, ayrılan parçaların distorsiyona uğramadan hassas bir şekilde birleştirilmesine yardımcı olurlar (Fornaini ve ark 2011). Seçilmiş ve ark (Secilmis ve ark 2012) ağız içi kaynak işlemi sırasında pulpadaki ısının tehlikeli boyutlara ulaşmaması için en az $2 \mathrm{~mm}$ kalınlığında dentin dokusunun olması gerektiğini vurgulamıştır.

\section{Akrilik rezinlerde lazer yüzey işlemi}

Genel olarak polimer materyaller zayıf adezyon kapasitesine sahiptirler. Bunun temelinde polimer maddelerinin çoğunun Islanabilirlik özelliklerinin zayıf olmasından kaynaklanır.

Bununla ilgili değişik çalışmalar protez kaidesinin yapısını değiştirerek yada bağlayıcı ajanlar kullanarak bu sorunu çözmeye çalışmışlardır (Wood ve ark 1993, Al-Athel ve ark 1995, Prosthodontics ve Session 1997). Lawrens ve Li (2001) yapmış oldukları bir çalışmada excimer lazerin polimetilmetakrilatın (PMMA) ıslanabilirliği üzerine anlamlı etkisinin olduğunu vurgulamıştır. Üşümez ve ark (2004) PMMA yüzeyini Nd:YAG lazer (15W, 2.6 $\mathrm{mm}$ demet çapı, enerji akısı 3500 joule $/ \mathrm{cm} 2$ ) ve kumlama $\left(250 \mu \mathrm{m} \mathrm{Al}_{2} \mathrm{O}_{3}, 1 \mathrm{~mm}\right.$ mesafe, $0.6 \mathrm{MPa}$ basınç altında) ile pürüzlendirdikleri bir çalışmada $\mathrm{Nd}$ :YAG lazerin istatistiksel olarak anlamlı fark oluşturduğunu bulmuşlardır. Tugut ve ark (2012) Er:YAG (3W, $300 \mathrm{mj}$, uzun süreli atım) lazer kullanımının PMMA ve yumuşak astar maddesi arasındaki bağlantının anlamlı derecede yükselttiğini bulmuşlardır.

\section{Endüstriyel lazer uygulamaları}

\subsection{Lazer sinterleme}

Illk defa 1980 yılında Dr. Joe Beaman tarafından geliştirilen selective laser sintering (SLS), küçük parçacıkların herhangi bir yapıştırıcı ajan olmaksızın yüksek enerji kaynağıyla uç uca birleştirilmesi olarak tanımlanır (Beaman ve ark 1997). Bu teknikle farklı metaller, polimerler, seramikler ve kompozit materyaller kullanılarak üç boyutlu hızlı prototipler yapılabilmektedir (Agarwala ve ark 1995). Direct metal lasersinteringler (DMLS), SLS den farklı olarak tek içerikli (singlecomponent) metal tozlar kullanır (Zong ve ark 1992). Her iki sistem içinde çoğunlukla yüksek güçlü (1-2 kw) $\mathrm{CO}_{2}$ lazer kullanılır. Bununla birlikte güç kaynağı olarak Nd:YAG lazer, Fiber lazer, disk lazer, Cu-vapour lazer kullanılabilmektedir (Glardon ve ark 2001, Hon ve Gill 2003). Lazer ile sinterlemede üretilen objenin kalitesi, kullanılan tozun özelliklerine, lazer parametrelerine (güç yoğunluğu, tarama hızı, tarama mesafesi) ayarlarına, objenin bulunduğu odadaki atmosfere bağlıdır. 


\section{SONUÇ}

Bu çalışmada protetik tedavilerde kullanılan lazerler ve bunların farklı materyaller üzerindeki etkileri tartışıldı. Ancak yapılmış çalışmaların birçoğu restoratif materyalin yüzey değişiklikleri ve bu değişikliklerin adeziv materyalleri nasıl etkileyeceği ile ilgiliydi. Gelecek çalışmalarda farklı lazerlerin materyalin fiziksel özellikleri üzerindeki etkileri incelenebilir.

\section{Protetik tedavilerde lazerlerin kullanımı}

Bu çalışmanın amacı lazer teknolojilerinin protetik tedavilere nasıl entegre edilebileceğini ve diş hekimliğinde kullanılan materyaller üzerindeki etkilerini tartışmaktır. $\mathrm{Bu}$ derleme, protetik tedavide kullanılan lazer çeşitlerini, lazer parametrelerini ve bunların materyaller olan etkileşimlerini inceleyen bilimsel çalışmaları kapsamaktadır.

\section{ANAHTAR KELIMELER}

Dental materyal, lazer, protetik tedavi, yüzey işlemi

\section{KAYNAKLAR}

Adachi M, Mackert J, Parry E, and Fairhurst C, 1990. Oxide adherence and porcelain bonding to titanium and Ti-6A1-4V alloy. Journal of dental research, 69, 1230-1235.

Agarwala M, Bourell D, Beaman J, Marcus $\mathrm{H}$, and Barlow J, 1995. Direct selective laser sintering of metals. Rapid Prototyping Journal, 1, 26-36.

Aida M, Hayakawa T, and Mizukawa K, 1995. Adhesion of composite to porcelain with various surface conditions. The Journal of prosthetic dentistry, 73, 464-470.

Akin $H$, Tugut F, Emine Akın G, Guney $U$, and BM, 2011. Effect of Er:YAG laser application on the shear bond strength and microleakage between resin cements and Y-TZP ceramics. Lasers Med Sci,"in press".

Akpinar YZ, Yavuz T, Aslan MA, Kepceoglu A, and Kilic HS, 2015. Effect of different surface shapes formed by femtosecond laser on zirconia-resin cement shear bond strength. Journal of Adhesion Science and Technology, 29, 149-157.
Akyıl MŞ, Uzun IH, and Bayındır F, 2010a. Bond strength of resin cement to yttrium-stabilized tetragonal zirconia ceramic treated with air abrasion, silica coating, and laser irradiation. Photomedicine and laser surgery, 28, 801-808.

Akyıl MŞ, Yılmaz A, Bayındır F, and Duymuş ZY, 2011. Microtensile bond strength of resin cement to a feldspathic ceramic. Photomedicine and laser surgery, 29, 197-203.

Akyıl MŞ, Yilmaz A, Karaalioğlu OF, and Duymuş ZY, 2010b. Shear bond strength of repair composite resin to an acid-etched and a laserirradiated feldspathic ceramic surface. Photomedicine and laser surgery, 28, 539-545.

Al-Athel MS, Jagger RG, and Jerolimov V, 1995. Bone strength of resilient lining materials to various denture base resins. The International journal of prosthodontics, 9, 167-170.

Aladag A, Comlekoglu ME, Dundar M, Gungor MA, and Artunc C, 2011. Effects of soldering and laser welding on bond strength of ceramic to metal. J Prosthet Dent, 105,28-34.

Atsu SS, Kilicarslan MA, and Kucukesmen HC, 2006. Effect of zirconium-oxide ceramic surface treatments on the bond strength to adhesive resin. The Journal of prosthetic dentistry,95, 430-436.

Attar N, Tam LE, and McComb D, 2003. Mechanical and physical properties of contemporary dental luting agents. The Journal of prosthetic dentistry, 89, 127-134.

Azzeh E, and Feldon PJ, 2003. Laser debonding of ceramic brackets: a comprehensive review. American journal of orthodontics and dentofacial orthopedics, 123, 79-83.

Barclay CW, Spence D, Laird WR, Marquis PM, and Blunt L, 2007. Micromechanical versus chemical bonding between $\mathrm{CoCr}$ alloys and methacrylate resins. Journal of Biomedical Materials Research Part B: Applied Biomaterials, 81, 351-357.

Beaman JJ, Barlow JW, Bourell DL, Crawford RH, Marcus HL, and McAlea KP, 1997. Solid freeform fabrication: a new direction in manufacturing. Kluwer Academic Publishers, Norwell, MA, 2061, 25-49.

Bertrand C, Le Petitcorps Y, Albingre L, and Dupuis $\mathrm{V}$, 2001. Prosthodontics: The laser welding technique applied to the non precious dental alloys procedure and results. British Dental Journal, 190, 255-257. 
Blatz M, 2003. Cementation of zirconium-oxide ceramic restorations. Practical procedures \& aesthetic dentistry: PPAD, 16, 14.

Blatz MB, Sadan A, Martin J, and Lang B, 2004. In vitro evaluation of shear bond strengths of resin to densely-sintered high-purity zirconium-oxide ceramic after long-term storage and thermal cycling. The Journal of prosthetic dentistry, 91, 356-362.

Borges GA, Sophr AM, De Goes MF, Sobrinho LC, and Chan DC, 2003. Effect of etching and airborne particle abrasion on the microstructure of different dental ceramics. The Journal of prosthetic dentistry, 89, 479-488.

Burke F, 1995. The effect of variations in bonding procedure on fracture resistance of dentin-bonded all-ceramic crowns. Quintessence international, 26, 293-300.

Cai Z, Shafer T, Watanabe I, Nunn ME, and Okabe $\mathrm{T}, 2003$. Electrochemical characterization of cast titanium alloys. Biomaterials, 24, 213-218.

Cavalcanti A, Foxton $R$, Watson $T$, Oliveira $M$, Giannini M, and Marchi G, 2009. Bond strength of resin cements to a zirconia ceramic with different surface treatments. Operative dentistry, 34, 280287.

Chai T, and Chou CK, 1998. Mechanical properties of laser-welded cast titanium joints under different conditions. The Journal of prosthetic dentistry, 79, 477-483.

Chen J-R, Oka K, Kawano T, Goto T, and Lchikawa $\mathrm{T}, 2010$. Carbon dioxide laser application enhances the effect of silane primer on the shear bond strength between porcelain and composite resin. Dental materials journal, 29, 731-737.

Creugers N, Snoek P, Van't Hof M, and KÄUYSER A, 1990. Clinical performance of resin-bonded bridges: a 5-year prospective study. Part III: failure characteristics and survival after rebonding. Journal of oral rehabilitation, $17,179-186$.

Da Silveira BL, Paglia A, Burnett Jr LH, Arai Shinkai RS, Eduardo CDP, and Spohr AM, 2005. Microtensile bond strength between a resin cement and an aluminous ceramic treated with Nd: YAG laser, Rocatec System, or aluminum oxide sandblasting. Photomedicine and laser surgery, 23, 543-548.

de Andrade AK, Feist IS, Pannuti CM, Cai S, Zezell DM, and De Micheli G, 2008. Nd:YAG laser clinical assisted in class II furcation treatment. Lasers in medical science, $23,341-347$. de Paula Eduardo C, Bello-Silva MS, Moretto SG, Cesar PF, and de Freitas PM, 2012. Microtensile bond strength of composite resin to glass-infiltrated alumina composite conditioned with $\mathrm{Er}, \mathrm{Cr}$ : YSGG laser. Lasers in medical science, 27, 7-14.

Demir N, Subaşı MG, and Ozturk AN, 2012. Surface roughness and morphologic changes of zirconia following different surface treatments. Photomedicine and laser surgery, 30, 339-345.

Dobberstein $H$, Schwarz A, Zuhrt $R$, and Tani $Y$, 1989. Laser processing of dental materials. Lasers in dentistry, 231-245.

Doğan GE, Demir T, and Orbak R, 2014. Periodontolojide Düşük Doz Lazer Uygulamaları. Journal of Marmara University Institute of Health Sciences, $4,1$.

Dominici JT, Sobczak KP, and Mitchell RJ, 1995. A Comparison of Infrared-and Torch-Soldering of Au$\mathrm{Pd}$ and Co-Cr Metal-Ceramic Alloys Using a HighFusing Solder. Journal of Prosthodontics, 4, 101110.

Eltas A, and Orbak R, 2013. Cerrahi olmayan periodontal tedavide Nd: YAG lazer kullanımı. Acta Odontologica Turcica, 30, 93.

Ersu B, Yuzugullu B, Ruya Yazici A, and Canay S, 2009. Surface roughness and bond strengths of glass-infiltrated alumina-ceramics prepared using various surface treatments. Journal of dentistry, 37 , 848-856.

Fasbinder DJ, 2010. Digital dentistry: innovation for restorative treatment. Compend Contin Educ Dent, 31, 2-11.

Feldon PJ, Murray PE, Burch JG, Meister M, and Freedman MA, 2010. Diode laser debonding of ceramic brackets. American Journal of Orthodontics and Dentofacial Orthopedics, 138, 458-462.

Fornaini C, Bertrand C, Bonanini M, Rocca JP, and Nammour S, 2009. Welding in the dental office by fiber-delivered laser: a new technique. Photomedicine and laser surgery , 27, 417-423.

Fornaini C, Passaretti F, Villa E, Rocca JP, Merigo E, Vescovi $\mathrm{P}$, Meleti M, Manfredi M, and Nammour S, 2011. Intraoral laser welding: ultrastructural and mechanical analysis to compare laboratory laser and dental laser. Lasers in medical science ,26, 415-420.

Foxton RM, Cavalcanti AN, Nakajima M, Pilecki P, Sherriff M, Melo L, and Watson TF, 2011. Durability of resin cement bond to aluminium oxide and zirconia ceramics after air abrasion and laser treatment. Journal of Prosthodontics , 20, 84-92. 
Fradeani M, and Redemagni M, 2002. An 11-year clinical evaluation of leucite-reinforced glassceramic crowns: a retrospective study. Quintessence international, 33, 503-510.

Galo R, Ribeiro RF, Rodrigues RCS, Pagnano VdO, and Mattos MdGCd, 2011. Effect of laser welding on the titanium ceramic tensile bond strength. Journal of Applied Oral Science, 19, 301-305.

Glardon R, Karapatis N, Romano V, and Levy G, 2001. Influence of $\mathrm{Nd}$ : YAG parameters on the selective laser sintering of metallic powders. CIRP Annals-Manufacturing Technology, 50, 133-136.

Gökçe B, Özpinar B, Dündar M, Cömlekoglu E, Sen $B$, and Güngör $M, 2007$. Bond strengths of allceramics: acid vs laser etching. Operative dentistry, 32, 173-178.

Guazzato M, Albakry M, Quach L, and Swain MV, 2005. Influence of surface and heat treatments on the flexural strength of a glass-infiltrated alumina/zirconia-reinforced dental ceramic. Dental Materials, 21, 454-463.

Guazzato M, Albakry M, Ringer SP, and Swain MV, 2004. Strength, fracture toughness and microstructure of a selection of all-ceramic materials. Part II. Zirconia-based dental ceramics. Dental materials, 20, 449-456.

Hautaniemi J, Herø H, and Juhanoja J, 1992. On the bonding of porcelain on titanium. Journal of Materials Science: Materials in Medicine, 3, 186191.

Hon K, and Gill T, 2003. Selective laser sintering of $\mathrm{SiC} /$ polyamide composites. CIRP AnnalsManufacturing Technology, 52, 173-176.

Jemt T, Henry P, Linden B, Naert I, Weber $\mathrm{H}$, and Bergstrom C, 2000. A comparison of laser-welded titanium and conventional cast frameworks supported by implants in the partially edentulous jaw: a 3-year prospective multicenter study. The International journal of prosthodontics, 13, 283.

Jemt T, and Linden B, 1991. Fixed implantsupported prostheses with welded titanium frameworks. The International journal of periodontics and restorative dentistry, 12, 177-184.

Jensen M, Sheth J, and Tolliver D, 1989. Etchedporcelain resin-bonded full-veneer crowns: in vitro fracture resistance. Compendium (Newtown, Pa.) , 10, 336-338, 40-41, 44-47.

Kara HB, Ozturk AN, Aykent F, Koc O, and Ozturk $B, 2011$. The effect of different surface treatments on roughnessand bond strength in low fusing ceramics. Lasers in medical science , 26, 599-604.
Kawano F, Dootz ER, Koran A, Craig RG, 1996. Bond Strength of Six Soft Denture Liners Processed Against Polymerized and Unpolymerized Poly (methyl Methacryiate). The International journal of prosthodontics, 10, 178-182.

Kern M, and Thompson V, 1994. Effects of sandblasting and silica-coating procedures on pure titanium. Journal of dentistry, 22, 300-306.

Kern M, and Wegner SM, 1998. Bonding to zirconia ceramic: adhesion methods and their durability. Dental Materials, 14, 64-71.

Kim JT, and Cho SA, 2009. The effects of laser etching on shear bond strength at the titanium ceramic interface. The Journal of prosthetic dentistry, 101, 101-106.

Kitasako Y, Burrow M, Katahira N, Nikaido T, and Tagami J, 2001. Shear bond strengths of three resin cements to dentine over 3 years in vitro. Journal of dentistry, 29, 139-144.

Kunt GE, Guler AU, Ceylan G, Duran I, Ozkan P, and Kirtiloglu T, 2012. Effects of Er:YAG laser treatments on surface roughness of base metal alloys. Lasers in medical science, 27, 47-51.

Lawrence J, and Li L, 2001. Modification of the wettability characteristics of polymethyl methacrylate (PMMA) by means of $\mathrm{CO} 2, \mathrm{Nd}$ : YAG, excimer and high power diode laser radiation. Materials Science and Engineering: A, 303, 142-149.

Lin MC, Lin SC, Wang YT, Hu SW, Lee TH, Chen LK, and Huang $\mathrm{HH}, 2007$. Fracture resistance of $\mathrm{Nd}$ :YAG laser-welded cast titanium joints with various clinical thicknesses and welding pulse energies. Dent Mater J, 26, 367-372.

Liu J, Watanabe I, Yoshida K, and Atsuta M, 2002. Joint strength of laser-welded titanium. Dental materials, 18, 143-148.

Lüthy $\mathrm{H}$, Loeffel $\mathrm{O}$, and Hammerle $\mathrm{CH}, 2006$. Effect of thermocycling on bond strength of luting cements to zirconia ceramic. Dental Materials, 22, 195-200.

Ma T, Marangoni RD, and Flint W, 1997. In vitro comparison of debonding force and intrapulpal temperature changes during ceramic orthodontic bracket removal using a carbon dioxide laser. American journal of orthodontics and dentofacial orthopedics, 111, 203-210.

Marinello C, Kerschbaum T, Heinenberg B, Hinz R, Peters S, Pfeiffer P, Reppel P, and Schwickerath H, 1987. Experiences with resin-bonded bridges and splints-a retrospective study. Journal of oral rehabilitation, 14, 251-260. 
Martínez-Insua A, da Silva Dominguez L, Rivera FG, and Santana-Penín UA, 2000. Differences in bonding to acid-etched or Er: YAG-laser-treated enamel and dentin surfaces. The Journal of prosthetic dentistry, 84, 280-288.

Miserendino LJ, 1995. The history and development of laser dentistry. Laser in Dentistry, 1st ed. Singapore, Quintessence, pp; 17.

Morford CK, Buu NC, Rechmann BM, Finzen FC, Sharma $A B$, and Rechmann P, 2011. Er: YAG laser debonding of porcelain veneers. Lasers in surgery and medicine, 43, 965-974.

Mundethu AR, Gutknecht N, and Franzen R, 2013. Rapid debonding of polycrystalline ceramic orthodontic brackets with an Er: YAG laser: an in vitro study. Lasers in medical science, 29, 1551 1556.

Murray AK, Attrill DC, and Dickinson MR, 2005. The effects of $\mathrm{XeCl}$ laser etching of $\mathrm{Ni}-\mathrm{Cr}$ alloy on bond strengths to composite resin: a comparison with sandblasting procedures. Dental materials: official publication of the Academy of Dental Materials, 21, 538-544.

NaBadalung DP, and Nicholls JI, 1998. Laser welding of a cobalt-chromium removable partial denture alloy. The Journal of prosthetic dentistry, 79, 285-290.

Oshida Y, and Hashem A, 1993. Titaniumporcelain system Part I: Oxidation kinetics of nitrided pure titanium, simulated to porcelain firing process. Bio-medical materials and engineering, 3, 185-198.

Osorio E, Toledano M, da Silveira BL, and Osorio $R, 2010$. Effect of different surface treatments on InCeram Alumina roughness. An AFM study. Journal of dentistry, 38, 118-122.

Oztoprak MO, Tozlu M, Iseri U, Ulkur F, and Arun T, 2012. Effects of different application durations of scanning laser method on debonding strength of laminate veneers. Lasers in medical science, 27, 713-716.

Östman PO, Hellman M, and Sennerby L, 2005. Direct implant loading in the edentulous maxilla using a bone density-adapted surgical protocol and primary implant stability criteria for inclusion. Clinical implant dentistry and related research, 7, 60-69.

Özcan M, Kerkdijk S, and Valandro LF, 2008. Comparison of resin cement adhesion to $\mathrm{Y}$-TZP ceramic following manufacturers' instructions of the cements only. Clinical Oral Investigations, 12, 279282.
Paranhos M, Burnett Jr LH, and Magne P, 2011. Effect Of Nd: YAG laser and $\mathrm{CO} 2$ laser treatment on the resin bond strength to zirconia ceramic. Quintessence international, 42, 79-89.

Piconi C, and Maccauro G, 1999. Zirconia as a ceramic biomaterial. Biomaterials, 20, 1-25.

Prasad S, and Monaco EA, 2009. Repairing an implant titanium milled framework using laser welding technology: a clinical report. J Prosthet Dent, 101:221-225.

Radovic I, Monticelli F, Goracci C, Vulicevic ZR, and Ferrari M, 2008. Self-adhesive resin cements: a literature review. J Adhes Dent, 10, 251-258.

Reyes M, Oshida Y, Andres C, Barco T, Hovijitra S, and Brown D, 2001. Titanium-porcelain system. Part III: Effects of surface modification on bond strengths. Bio-medical materials and engineering, 11, 117-136.

Rickabaugh JL, 1994. Laser debonding of ceramic orthodontic brackets. American Journal of Orthodontics and Dentofacial Orthopedics, 105, 214.

Santos M, Acciari H, Vercik L, and Guastaldi A, 2003. Laser weld: microstructure and corrosion study of $\mathrm{Ag}-\mathrm{Pd}-\mathrm{Au}-\mathrm{Cu}$ alloy of the dental application. Materials Letters, 57, 1888-1893.

Sarp ASK, and Gülsoy M, 2011. Ceramic bracket debonding with ytterbium fiber laser. Lasers in medical science, 26, 577-584.

Secilmis A, Bulbul M, and Usumez A, 2012. Temperature rise during laser welding on base metal castings. Lasers Med Sci, "in press".

Shimakura M, Yamada S, Takeuchi M, Miura K, and Ikeyama J, 2009. Influence of irradiation conditions on the deformation of pure titanium frames in laser welding. Dental materials journal, 28, 243-247.

Shiu P, De Souza-Zaroni WC, Eduardo CdP, and Youssef MN, 2007. Effect of feldspathic ceramic surface treatments on bond strength to resin cement. Photomedicine and laser surgery, 25, 291296.

Sjögren G, Andersson M, and Bergman M, 1988. Laser welding of titanium in dentistry. Acta Odontologica, 46, 247-253.

Sorensen JA, Engelman MJ, Torres TJ, and Avera SP, 1990. Shear bond strength of composite resin to porcelain. The International journal of prosthodontics, 4, 17-23. 
Spohr AM, Borges GA, Júnior LHB, Mota EG, and Oshima HMS, 2008. Surface modification of InCeram Zirconia ceramic by Nd: YAG laser, Rocatec system, or aluminum oxide sandblasting and its bond strength to a resin cement. Photomedicine and laser surgery , 26, 203-208.

Stewart GP, Jain P, and Hodges J, 2002. Shear bond strength of resin cements to both ceramic and dentin. The Journal of prosthetic dentistry , 88, 277-284.

Subaşı MG, and Inan O, 2011. Evaluation of the topographical surface changes and roughness of zirconia after different surface treatments. Lasers Med Sci "in press".

Suzuki Y, Ohkubo C, Abe M, and Hosoi T, 2004 Titanium removable partial denture clasp repair using laser welding: a clinical report. The Journal of prosthetic dentistry, 91, 418-420.

Tocchio RM, Williams PT, Mayer FJ, and Standing KG, 1993. Laser debonding of ceramic orthodontic brackets. American Journal of Orthodontics and Dentofacial Orthopedics, 103, 155-162.

Tugut F, Akin H, Mutaf B, Akin GE, and Ozdemir AK, 2012. Strength of the bond between a silicone lining material and denture resin after Er:YAG laser treatments with different pulse durations and levels of energy. Lasers in medical science, 27, 281-285.

Ural Ç, KalyoncuoGlu E, and Balkaya V, 2012. The effect of different power outputs of carbon dioxide laser on bonding between zirconia ceramic surface and resin cement. Acta Odontologica Scandinavica, 70, 541-546.

Ural Ç, Külünk T, Külünk $S$, and Kurt M,2010. The effect of laser treatment on bonding between zirconia ceramic surface and resin cement. Acta Odontologica Scandinavica, 68, 354-359.

Usumez A, Handemirci N, Koroglu BY, Simsek I, Parlar O, and Sari T, 2012.Bond strength of resin cement to zirconia ceramic with different surface treatments. Lasers Med Sci, "in press".

Usumez A, Inan O, and Aykent F, 2004. Bond strength of a silicone lining material to aluminaabraded and lased denture resin. Journal of Biomedical Materials Research Part B: Applied Biomaterials, 71, 196-200.

Wang RR and Fenton A, 1996. Titanium for prosthodontic applications: a review of the literature. Quintessence Int, 27, 401-408.

Wiskott HA, Doumas T, Scherrer SS, Susz C, and Belser UC, 2001. Microstructures of brazings and welds using grade 2 commercially pure titanium. International Journal of Prosthodontics, 14, 40-47.
Wolfart M, Lehmann F, Wolfart S, and Kern M, 2007. Durability of the resin bond strength to zirconia ceramic after using different surface conditioning methods. Dental Materials, 23, 45-50.

Wood WE, Johnson DL, and Duncanson MG, 1993. Variables Affecting Silicone-Polymethyl Methacrylate Interfacial Bond Strengths. Journal of Prosthodontics, 2, 13-18.

Wulfes $\mathrm{H}, 2003$. Precision milling and partial denture constructions. Academia Dental, Lakewood.

Yilmaz A, Akyil MS, and Hologlu B, 2011. The effect of metal primer application and $\mathrm{Nd}$ :YAG laser irradiation on the shear-bond strength between polymethyl methacrylate and cobalt-chromium alloy. Photomed Laser Surg, 29, 39-45.

Yilmaz H, Aydin C, and Gul BE, 2007. Flexural strength and fracture toughness of dental core ceramics. The Journal of prosthetic dentistry, 98, 120128.

Zong G, Wu Y, Tran N, Lee I, Bourell D, Beaman J, and Marcus $H, 1992$. Direct selective laser sintering of high temperature materials. Solid Freeform Fabrication Symposium Proceedings, pp. 72-85. The University of Texas at Austin.

Zupancic R, Legat A, and Funduk N, 2006. Tensile strength and corrosion resistance of brazed and laser-welded cobalt-chromium alloy joints. The Journal of prosthetic dentistry, 96, 273-282.

\section{Yazışma Adresi:}

Yrd. Doç. Dr. Yusuf Ziya AKPINAR

Abant Izzet Baysal Üniversitesi

Diş Hekimliği Fakültesi

Protetik Diş Tedavisi AD

14280 Gölköy / BOLU

Tel: +90 (374) $2541000 / 8370$

Faks: +90 (374) 2541000

E-mail: yza61@yahoo.com 